\title{
Perda de peso em judocas no período pré-competitivo: uma revisão da literatura
}

\author{
Gustavo de Araujo ${ }^{a}$, Danilo Aparecido Foster ${ }^{\mathrm{a}}$, Leonardo Emmanuel de Medeiros Lima ${ }^{\mathrm{b}}$ (D), Henrique

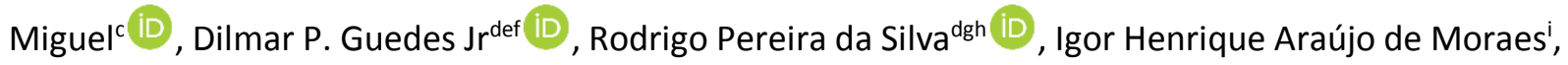 \\ Roberto Moriggi Jraci (iD
}

aFaculdade de Santa barbara D'Oeste (UNIESP), Santa barbara D'Oeste, SP, Brasil.

bUniversidade São Judas Tadeu, (SÃO JUDAS), São Paulo, SP, Brasil.

'Centro Universitário de Jaguariúna (EAD UNIFAJ - UNIEDUK), Jaguariúna, SP, Brasil.

dUniversidade Metropolitana de Santos (UNIMES), Santos, SP, Brasil.

eUniversidade Santa Cecilia (UNISANTA), Santos, SP, Brasil.

${ }^{f}$ Centro de Treinamento Fisiologia do Exercício e Treinamento (CEFIT), São Paulo, SP, Brasil.

gFaculdade Praia Grande (FPG), Praia Grande, SP, Brasil.

hUniversidade Federal de São Paulo (UNIFESP), Santos, SP, Brasil.

'Centro Universitário Max Planck (UNIMAX), Indaiatuba, SP Brasil.

RESUMO O judô tem grande ênfase no autoconhecimento. As competições são essenciais para o desenvolvimento no esporte, os lutadores na busca de se beneficiar durante uma competição recorrem a várias estratégias de perda de peso, para assim, se classificar em uma categoria desejada. Os competidores acreditam obter vantagem sobre seus adversários lutando em uma categoria abaixo. Contudo essa suposta vantagem para ser classificado dentro da categoria de peso especifica exige sacrifícios e possíveis prejuízos. Sendo assim, o presente estudo tem como objetivo analisar pesquisas recentes sobre a perda de peso no período pré-competitivo em atletas de judô e seus efeitos adversos à saúde e ao desempenho. A pesquisa se caracteriza como uma revisão de literatura narrativa com busca em bases de dados e sites de busca como Google Acadêmico, Scielo, Capes e PubMed. Os achados apresentados no presente estudo mostram que na tentativa de obter vantagens, lutando com uma maior massa corporal em uma certa categoria, os lutadores utilizam da redução rápida de peso, perdendo até $5 \%$ da massa corporal em curto período de tempo com uma recuperação de até no máximo 24 horas até o combate. Entre os procedimentos mais utilizados estão: a desidratação e a restrição calórica. Tais métodos descritos acarretam a debilitação física e comprometimentos para a saúde.

$$
\text { PALAVRAS-CHAVE judô, pré-competição, perda de peso }
$$

Aceito 28 de janeiro de 2021 Publicado online 29 de março de 2021

Cite este artigo:

Araujoet al. (2021) Perda de peso em judocas no período pré-competitivo: uma revisão da literatura. Multidisciplinary Reviews 4: e2021007.DOI: 10.29327/multi.2021007.

\section{Weight loss in judo athletes in the pre-competitive period: a literature review}

ABSTRACT Judo has a great emphasis on self-knowledge. Competitions are essential for developing the sport, fighters seeking to benefit during a competition resort to various weight-loss strategies to classify themselves in the desired category. Competitors believe they have an advantage over their opponents by fighting in a category below. However, this supposed advantage to be classified within the specific weight category requires sacrifices and possible losses. Therefore, this study aims to analyze recent research on weight loss in judo athletes' pre-competitive period and its adverse effects on health and performance. The research reviews narrative literature searching in databases and search sites such as Google Scholar, Scielo, Capes, and PubMed. Within this conception, the study will be based on the damage to the athletes' health and development. The findings presented in the present study show that in an attempt to obtain 
advantages, struggling with greater body mass in a specific category, fighters use rapid weight reduction, losing up to $5 \%$ of body mass in a short time with a recovery of up to a maximum of 24 hours until combat. Among the most used procedures are dehydration and caloric restriction. Such methods described lead to physical weakness and health compromises.

KEYWORDS: weight-loss, pre-competition, judo

\section{Introdução}

O judô é uma das modalidades de esportes de combate, que possui como característica o embate entre dois oponentes, buscando a vitória dentro das regras especificas da luta (Carazzato et al 1996). Para buscar o equilíbrio entre os combates, os atletas são divididos por categorias tanto masculina quanto feminina, minimizando as diferenças de força e velocidade, equiparando também a massa corporal, graduação e idade, permitindo assim uma disputa mais justa (Artioli et al 2006; Franchini 2010).

Com intuito de se beneficiar na competição, atletas recorrem da perda rápida de peso até momentos antes da pesagem oficial e o recuperam após a pesagem para ganhar vantagens e assim competindo com seu estado de peso normal, teoricamente, para ter uma vantagem lutando em categorias mais leves contra oponentes mais fracos (Steen 1990). Para redução da massa corporal em um curto espaço de tempo, existem várias estratégias, sendo muitas das vezes extremamente severas, procedimentos esses como a restrição da ingestão de alimentos e de líquidos, o aumento do volume e intensidade do treinamento em locais com a temperaturas elevadas, o uso de roupas exageradas, como kimonos, roupas de plástico ou borracha para indução do suor, a utilização de sauna, o uso de medicamentos como laxantes e diuréticos e algumas vezes chegando até mesmo a indução ao vômito (Artioli et al 2010a).

A perda de massa corporal é culturalmente considerada como uma parte essencial para o judô esportivo, visto que esta modalidade é classificada por categorias de peso (Sundgot-Borgen et al 2013). Esses procedimentos de perda de peso permanecem sendo realizados, podendo estar atribuídos a alguns aspectos como a inexistência de conhecimento por parte dos atletas e treinadores sobre os riscos com relação ao desempenho e a sua saúde, colocando o desejo de vencer a qualquer custo (Santos et al 2018). Por outro lado, Graves et al (1991) sustentam, que parte dos atletas e treinadores possuem bons conhecimentos, todavia, vale salientar a falta de relação entre essas competências com esses costumes e estratégias adequadas. Desta forma, o presente estudo busca apresentar o atual corpo de pesquisa sobre redução de peso no judô, incluindo prevalência, práticas comuns, tempo da redução do peso, quantidade do peso perdido pré-competição, tempo de recuperação após a pesagem, estratégias utilizadas para redução do peso bem como os efeitos da redução rápida na saúde e no desempenho.

\section{Métodos}

O método tem por finalidade conduzir a pesquisa até o objeto de estudo, caracterizado como uma revisão de literatura narrativa, onde se desenvolve a pesquisa em buscas bibliográficas em base de dados e buscadores via online, utilizadas Google Acadêmico, Scielo, Capes e PubMed.

\section{Tempo e quantidade de peso perdido até a pesagem oficial}

A massa corporal é uma medida essencial, que pode variar muito de acordo com a idade e gênero (Luque et al 2016). Por esse mesmo motivo no judô competitivo, os atletas são divididos por categorias de peso, idade, gênero e graduação. Estas subdivisões são utilizadas para minimizar as diferenças. Quanto a massa corporal, essa influência diretamente nas manifestações de força, velocidade e resistência dos movimentos e golpes aplicados (Artioli et al 2006).

A rápida perda de peso no judô é uma atitude comum entre seus praticantes, independente do sexo (Góes et al 2018). Os judocas utilizam de manobras para redução do peso no intuito de ter uma certa vantagem no combate contra um oponente mais leve e fraco, lutando em uma categoria abaixo do seu peso real (Artioli et al 2016). Os atletas 
de judô no Brasil têm dificuldades em manter o peso para lutar na categoria desejada e, por esse motivo, reduzem uma parte dele, até dias antes da competição (Artioli et al 2007).

Artioli et al (2010), buscaram investigar o tempo de redução até a pesagem oficial, onde foram feitas coletas de dados em pequenos torneios, regionais, estaduais em São Paulo, bem como competições de nível nacional, internacional e mundial realizadas no Rio de Janeiro. Contando com uma amostra de 822 atletas de várias categorias e diferentes regiões do Brasil, sendo 607 do gênero masculino e 215 do gênero feminino. Foi visto que 37.2\% da amostra relatou perder o peso de 3 dias a horas antes da pesagem. A média da redução de peso foi de cerca de $5 \%$ da massa corporal.

Fabrini et al (2010) realizaram um estudo com atletas de judô de diferentes níveis e categorias no estado de minas gerais, onde do total da amostra foi 125 atletas, sendo 105 masculinos e 20 femininos. Os resultados demonstraram que $\mathbf{7 7 , 1 \%}$ dos homens e $55 \%$ das mulheres relataram reduzir a massa corporal na semana précompetitiva e que os homens reduzem em média $4 \%$ e as mulheres a $2 \%$ da massa corporal nesse mesmo período.

Em outro estudo realizado por Olívio et al (2015), em uma cidade do interior de São Paulo, realizada com 9 atletas federados de judô, com idades entre 14 a 22 anos de ambos os sexos e diferentes categorias, relataram que os atletas perderam peso de forma brusca dias ou até mesmo horas antes das competições, com a maioria dos participantes chegando a perder até $1,5 \%$ da massa corporal de um dia para outro.

Em uma outra pesquisa realizado por Brito et al (2012) de cunho descritivo, no estado de Minas Gerais, dentro das academias e clubes, reunindo vários lutadores de diferentes modalidades de combate. Tendo uma amostra final do judô com 145 homens, relatando um percentual de perda de peso no período pré-competitivo em média de 8,5 $\pm 4,2 \%$ da massa corporal, perdida rapidamente a partir de uma semana antes da pesagem oficial.

Santos et al (2018) em um estudo mais recente realizado com 31 atletas sendo 20 homens e 11 mulheres, com idade entre 20 a 30 anos, faixas pretas e marrons. Foi visto que uma grande parte da amostra fez a redução em um período de 5 dias antecedentes a pesagem oficial e em média a redução da massa corporal foi de $3 \%$, sendo 15 do gênero masculino e 9 do gênero feminino.

Sendo assim, os estudos mostram que, há indícios que grande parte dos judocas reduzem o peso rapidamente antes das competições. Em relação ao tempo da redução descrito pelos autores, foi cerca de sete dias ou mesmo momentos antes. Pode-se observar então que essa prática é usual, mesmo tendo potencial negativo para o desempenho e saúde (Santos et al 2018), principalmente quando grandes quantidades de peso são perdidas, tema abordado no tópico subsequente. Desta forma, relatado nos estudos, os lutadores perderam até $5 \%$ da massa corporal em curto período de tempo, as vezes em menos de 24 horas de modo severo, o que pode ser prejudicial ao desempenho.

\section{Estratégias utilizadas para redução do peso}

São inúmeras estratégias, desde exercícios de grande intensidade, a restrição hídrica, utilização de diuréticos, corrida com utilização de agasalhos, treinos de judô com mais veemência, a redução drástica da alimentação e saunas (Artioli et al 2010a). Em um estudo de Mazzoccante et al (2013) realizado com 60 lutadores das categorias Juvenil, Juniores Sêniores e Master de nível nacional, apontaram que há uma diferença em relação a preferência dos atletas em realizar a estratégia de perda de peso rápida. Na categoria juvenil, as mais usadas são correr e reduzir a alimentação; atletas juniores preferem reduzir a alimentação; os seniores destacam-se por fazer exercícios mais intensos; e os judocas da categoria máster preferem treinar judô mais intensamente e reduzir a alimentação.

Os possíveis efeitos da rápida perda de peso utilizando de métodos agressivos ainda permanecem em controversos, independentemente do método adotado para a redução. Assim como todos protocolos adotados devem surgir alguns efeitos de forma positiva ou negativa, o mais importante no momento é não afetar o desempenho e a saúde dos judocas.

\section{Efeitos da redução rápida do peso na saúde e no desempenho}

Estar em boas condições físicas e mentais para lutar é o que todos atletas almejam após realizar os métodos de redução de peso. Quanto ao seu desempenho, por já terem sofrido um grande stress para atingir o seu peso ideal na 
categoria, é de se questionar se o protocolo adotado para perda de peso irá afetar sua performance durante a competição.

Sendo assim, os autores Kraft et al (2013) mostraram que o desempenho pode ser prejudicado pela exposição ao calor e/ou desidratação do atleta. Nesse aspecto, os atletas quando alcançam níveis relativamente moderados de desidratação, reduzem o volume plasmático, desencadeando uma redução do débito cardíaco e volume de ejeção, limitando a oxigenação muscular, causando diminuição no desempenho relacionado com a diminuição da resistência, aumento da fadiga, alteração da capacidade de termo regulação (temperatura), redução da motivação e aumento da taxa de esforço.

Já para Mendes et al (2013), que avaliaram o efeito da redução rápida de peso através de exercícios intensos e restrição hídrica, seguida por um período de recuperação de 4 horas, sobre o desempenho, observaram que não houve diferenças significativas na potência média, na potência pico ou no trabalho total realizado, demonstrando que o desempenho não é prejudicado pela redução rápida de peso, quando existe um período de recuperação entre a pesagem e o início dos testes, independentemente se os atletas são familiarizados ou não com as práticas de perda de peso. Quanto a percepção dos atletas durante as lutas e sobre seu desempenho, Mazzoccante et al (2013) relataram em seu estudo feito com 33 judocas de ambos os sexos, que $48 \%$ sentiram uma queda no seu rendimento total durante a luta, bem como uma mudança de humor e desmotivação durante a competição.

Com isso podemos sugerir que há a redução de peso, visto que dois dos três autores destacados relatam essa redução do desempenho, entretanto quanto menor o peso perdido, um tempo de recuperação adequado e uma reidratação correta, o desempenho do atleta pode não sofrer uma grande queda. Dado a circunstância é importante sabermos do tempo de recuperação da redução do peso perdido, o qual é determinante na recuperação do desempenho, que será abordado no tema a seguir.

\section{Tempo de recuperação do peso após a pesagem oficial}

A um período entre a pesagem e o início da luta, favorecendo tais práticas de redução de peso. O que gera a oportunidade para que os atletas façam a reposição de nutrientes e líquidos, para assim atenuar os efeitos negativos da perda rápida de peso e recuperar boa parte ou o total de peso perdido (Franchini et al 2012).

Segundo Artioli et al (2011), com 117 atletas de judô das classes juvenil, júnior e sênior, todos do sexo masculino, demostraram que o período médio entre a pesagem e o início das lutas foi de aproximadamente 4 horas, onde a maior parte dos atletas tinham aproximadamente 2,5 - 5 horas como tempo de recuperação para o combate.

Em um outro estudo Mendes et al (2013), investigaram 21 atletas de combate todos do sexo masculino, sendo 12 atletas de judô. De acordo com os resultados da pesquisa, o tempo após a pesagem foi de no máximo de 4 horas para recuperar, se alimentando e reidratando, o que corrobora com o estudo de Artioli et al (2011). Logo após a pesagem da competição os lutadores buscam rapidamente restabelecer o seu peso habitual (Matthews et al 2018).

Reale et al (2016) investigou 86 atletas de ambos gêneros, durante o open internacional de judô na Austrália. Os sujeitos foram pesados duas vezes, sendo a primeira na pesagem oficial e segunda uma hora antes de sua primeira luta (15-20 horas depois), que representa um período de 15 a 20 horas a partir da pesagem oficial. Desta maneira os estudos relatam um tempo de aproximadamente 2 a 20 horas entre a pesagem e o início da competição.

Como uma forma de diminuir com as práticas de redução de peso de forma brusca que pode vir a prejudicar a saúde do atleta, a federação Brasileira de judô já adotou em seu regulamento de competição o sorteio de atletas para se pesar novamente 45 minutos antes de se iniciar os combates, isso vale para as classes sub- 18 , sub- 21 e sênior. A pesagem começará imediatamente após o sorteio e terá duração de 30 minutos. A lista de atletas sorteados será divulgada na área de aquecimento/concentração dos competidores. Os atletas sorteados devem comparecer a pesagem e seu peso não poderá exceder a $5 \%$ do limite de sua respectiva categoria, isso ainda estando dentro do seu peso perdido (RNE-CBJ 2019). Segundo Fortes et al (2017) em um período de 2 semanas, antecedentes a pesagem com uma redução de $10 \%$ da massa corporal pode ocorrer a diminuição do desempenho.

\section{Discussão}


A perda de peso aguda no período pré-competitivo vem amplamente sendo utilizada por muitos atletas de judô. Visto que os judocas adotam tais estratégias a fim de adquirir vantagem sobre seus oponentes, competindo na categoria abaixo do seu peso habitual. Os achados na literatura, apontados no presente estudo mostram que por dificuldades em manter o peso, os lutadores utilizam da redução rápida de peso, perdendo até $5 \%$ da massa corporal em curto período de tempo com uma recuperação de até no máximo 24 horas até o combate.

Entre os procedimentos mais utilizados estão: a desidratação e a restrição calórica. Tais métodos descritos acarretam a debilitação física e mental. Uma série de prejuízos para o desempenho e principalmente a saúde. Danos agudos e crônicos, uma vez que esses procedimentos irão se repetir várias e várias vezes ao longo de sua carreira.

Uma limitação dos estudos investigados e comparar a perda rápida de peso e o sucesso competitivo, devido principalmente à imprevisibilidade e interdependência das ações dos judocas, não há meios de medir diretamente o desempenho na modalidade. Atualmente, já existem estratégias a fim de minimizar a prevalência e magnitude das práticas de redução de peso, que podem ser importantes no futuro. No entanto, há uma escassez de dados sobre sua eficácia, o que deve ser investigada por pesquisas futuras.

\section{Considerações finais}

Os achados apresentados no presente estudo mostram que na tentativa de obter vantagens, lutando com uma maior massa corporal em uma certa categoria, os lutadores utilizam da redução rápida de peso, perdendo até $5 \%$ da massa corporal em curto período de tempo com uma recuperação de até no máximo 24 horas até o combate. Entre os procedimentos mais utilizados estão: a desidratação e a restrição calórica. Tais métodos descritos acarretam a debilitação física e comprometimentos para a saúde.

\section{Declaração de conflito de interesse}

Os autores afirmam não ter conflitos de interesses financeiros ou outros (comerciais, pessoais ou políticos) relacionados ao tema e ao desenvolvimento do artigo submetido para publicação.

\section{Referências}

Artioli GG, Gualano B, Franchini E, Scagliusi FB, Takesian M, Fuchs M, Lancha Jr AH (2010) Prevalence, magnitude, and methods of rapid weight loss among judo competitors. Med Sci Sports Exerc 42:436-442.

Artioli GG, Saunders B, Iglesias RT, Franchini E (2016) It is time to ban rapid weight loss from combat sports. Sports Medicine 46:15791584.

Artioli GG, Franchini E, Solis MY, Fuchs M, Takesian M, Mendes SH, Lancha Junior AH (2011) Tempo de recuperação entre a pesagem e o início das lutas em competições de judô do Estado de São Paulo. Revista Brasileira de Educação Física e Esporte 25:371-376.

Artioli GG, Franchini E, Lancha Junior AH (2006) Perda de peso em esportes de combate de domínio: revisão e recomendações aplicadas. Revista Brasileira de Cineantropometria \& Desempenho Humano 8:2.

Carazzato JG, Cabrita H, Castropil W (1996) Repercussão no aparelho locomotor da prática do judô de alto nível. Rev Bras Ortop 31:957-68.

De Góes GH, Da Silva Azzi A, Saldanha RP, Garlipp DC (2018) Prevalência e métodos de perda de peso rápida em atletas de judô. Revista Brasileira de Nutriçao Esportiva 12:1005-1010.

Dos Santos CA, Da Cruz FL, Amadio MB, Do Carmo EC (2018) Conhecimento de atletas de judô sobre os problemas decorrentes da desidratação no processo de perda de peso no período pré-competitivo. Revista Brasileira de Nutrição Esportiva 12:276-281.

Fabrini SP, Brito CJ, Mendes EL, Sabarense CM, Marins JCB, Franchini E (2010) Práticas de redução de massa corporal em judocas nos períodos pré-competitivos. Revista Brasileira de Educação Física e Esporte 24:165-177, 2010.

Fortes LS, Costa BD, Paes PP, Cyrino ES, Vianna JM, Franchini E (2017) Effect of rapid weight loss on physical performance in judo athletes: is rapid weight loss a help for judokas with weight problems?. International Journal of Performance Analysis in Sport 17:763773.

Franchini E (2010) Judô: Desempenho Competitivo. 2a edição. Manole.

Franchini E, Del Vecchio FB (2011) Estudos em modalidades esportivas de combate: estado da arte. Revista brasileira de educação física e esporte 25:67-81. 
Graves KL, Farthing MC, Smith SA, Turchi JM (1991) Nutrition training, attitudes, knowledge, recommendations, responsibility, and resource utilization of high school coaches and trainers. Journal of the American Dietetic Association 91:321-324.

Matthews JJ, Stanhope EN, Godwin MS, Holmes ME, Artioli GG (2019) The magnitude of rapid weight loss and rapid weight gain in combat sport athletes preparing for competition: A systematic review. International journal of sport nutrition and exercise metabolism 29:441-452.

Mendes SH, Tritto AC, Guilherme JPL, Solis MY, Vieira DE, Franchini E, Artioli GG (2013) Effect of rapid weight loss on performance in combat sport male athletes: does adaptation to chronic weight cycling play a role? British journal of sports medicine 47:1155-1160.

Miyamoto AH, De Almeida BS, Ribeiro K, Vasconcelos N, Belmonte S, Alvarenga M (2018) Avaliação do consumo alimentar pré e pós competição de Judô. RBNE-Revista Brasileira De Nutrição Esportiva 12:178-184.

Morales J, Ubasart C, Solana-Tramunt M, Villarrasa-Sapiña I, González LM, Fukuda D, Franchini E (2018) Effects of rapid weight loss on balance and reaction time in elite judo athletes. International journal of sports physiology and performance 13:1371-1377.

Olivio AB, Junior JAO, De Cillo ENP, Faleiros PB (2015) Análise comportamental de estratégias de redução brusca de peso de judocas em período de competição. Revista Brasileira de Terapia Comportamental e Cognitiva 17:4-21.

Reale R, Cox GR, Slater G, Burke LM (2016) Regain in body mass after weigh-in is linked to success in real life judo competition. International journal of sport nutrition and exercise metabolism 26:525-530.

Steen VD, Costantino JP, Shapiro AP, Medsger Jr TA (1990) Outcome of renal crisis in systemic sclerosis: relation to availability of angiotensin converting enzyme (ACE) inhibitors. Annals of internal medicine 113:352-357.

Sundgot-Borgen J, Meyer NL, Lohman TG, Ackland TR, Maughan RJ, Stewart AD, Müller W (2013) How to minimise the health risks to athletes who compete in weight-sensitive sports review and position statement on behalf of the Ad Hoc Research Working Group on Body Composition, Health and Performance, under the auspices of the IOC Medical Commission. British journal of sports medicine 47:1012-1022.

Torres-Luque G, Hernández-García R, Escobar-Molina R, Garatachea N, Nikolaidis PT (2016) Physical and physiological characteristics of judo athletes: An update. Sports 4:1. 